\title{
Colombia y su política de fronteras: retos y perspectivas a la luz del litigio con Nicaragua ante la Corte Internacional de Justicia*
}

\section{Diana María eAngulo Cardona* María Isabel Cadrazco Saavedra* Alexander Pinzón Flórez ${ }^{* * *}$}

\section{Para citar este artículo}

Angulo, D., Cadrazco, M. \& Pinzón, A. (2021). Colombia y su política de fronteras: retos y perspectivas a la luz del litigio con Nicaragua ante la Corte Internacional de Justicia. Vía luris, 30, 95110. DOI: https://doi.org/10.37511/viaiuris.n30a5

\section{RESUMEN}

A lo largo de 210 años de vida republicana, el Estado colombiano ha perdido y cedido parte del territorio nacional con relación al tamaño del Virreinato de la Nueva Granada. De 1.572.248 $\mathrm{km}^{2}$ de territorio que pertenecieron al Virreinato, tan solo $1.147 .748 \mathrm{~km}^{2}$ corresponden al actual territorio nacional. Es decir, el país ha perdido unos $504.500 \mathrm{~km}^{2}$ o $579.500 \mathrm{~km}^{2}$ si se suma el territorio perdido a causa del litigio marítimo con Nicaragua ${ }^{1}$. Ante la falta de políticas públicas de Estado que respondieran con efectividad y eficacia a la defensa de los intereses nacionales en el territorio insular colombiano, el fallo de la Corte Internacional de Justicia de La Haya de 2012 frente a la demanda interpuesta por Nicaragua contra Colombia, en reclamo de soberanía sobre el Archipiélago, sumó $75.000 \mathrm{~km}^{2}$ más a la pérdida territorial histórica que ha sufrido el país. El presente artículo analiza los retos de la política de fronteras de Colombia después de la Constitución de 1991, el contexto social y político en el que los últimos dos gobiernos nacionales han implementado dichas políticas en la isla y la incidencia que el fallo de la Corte Internacional de Justicia (CIJ) tuvo en la redefinición no solo de la frontera marítima colombo-nicaragüense, sino también de toda la política de fronteras de Colombia.

1 Datos registrados en el libro "La llegada del dragón: ¿̇alló La Haya?" de los autores Noemí Sanín Posada y Miguel Ceballos Arévalo de 2013.

* Este artículo es producto del proyecto de investigación, Colombia y su política de fronteras: Retos y perspectivas a la luz del litigio con Nicaragua ante la Corte Internacional de Justicia, del Grupo de investigación, Centro de Análisis Políticos Nacionales e Internacionales CAPNI. Periodo 2020. Bogotá, Colombia.

** Magíster en Estudios Políticos e Internacionales del Rosario, Especialista en Administración Pública de la Escuela Superior de Administración Pública y Politóloga de la Universidad del Cauca. Docente de tiempo completo del Programa de Gobierno y Relaciones Internacionales de la Universidad La Gran Colombia. Bogotá, Colombia. Correo electrónico de contacto: diana. angulo@ugc.edu.co ORCID: https://orcid.org/0000-0002-5293-1988

*** Magíster en Relaciones Internacionales e Internacionalista de la Universidad del Norte. Docente investigadora del Programa de Gobierno y Relaciones Internacionales de la Universidad La Gran Colombia. Bogotá, Colombia. Correo electrónico de contacto: maria.cadrazco@ugc.edu.co. ORCID: https://orcid.org/0000-0002-7366-4006.

**** Magíster en Política y Relaciones Internacionales y Profesional en Filosofía y Humanidades de la Universidad Sergio Arboleda. Docente investigador del Programa de Gobierno y Relaciones Internacionales de la Universidad La Gran Colombia. Bogotá, Colombia. Correo electrónico de contacto: jaiber.pinzon@ugc.edu.co ORCID: https://orcid.org/0000-0002-0305-5117
DOI: https://doi.org/10.37511/viaiuris.n30a5

Creative Commons Atribución-NoComercial-Compartirlgual 4.0 Internacional (CC BY-NC-SA 4.0).

\section{(c) (1) (8)}

Palabras clave

Política de frontera, Colombia, Nicaragua, diferendo limítrofe, derecho internacional, Corte Internacional de Justicia. 


\title{
Colombia and its border policy: challenges and perspectives in light of the litigation with Nicaragua before the International Court of Justice
}

\author{
Diana María Angulo Cardona \\ María Isabel Cadrazco Saavedra \\ Alexander Pinzón Flórez.
}

\begin{abstract}
Throughout 210 years of republican life, the Colombian State has lost and ceded part of the national territory in relation to the size of the Viceroyalty of New Granada. Of 1,572,248 km2 of territory that belonged to the Viceroyalty, only $1,147,748 \mathrm{~km} 2$ correspond to the current national territory. In other words, the country has lost about $504,500 \mathrm{~km} 2$ or $579,500 \mathrm{~km} 2$ if the territory lost due to the maritime litigation with Nicaragua is added. Given the lack of public State policies that responded effectively and efficiently to the defense of national interests in the Colombian island territory, the 2012 ruling of the International Court of Justice in The Hague against the lawsuit filed by Nicaragua against Colombia in claim of sovereignty over the Archipelago, added 75,000 km2 more to the historical territorial loss that the country has suffered. This article analyzes the challenges of Colombia's border policy after the 1991 Constitution, the social and political context in which the last two national governments have implemented these policies on the Island, and the impact that the ICJ ruling had on the redefinition not only of the Colombian-Nicaraguan maritime border, but also of the entire border policy of Colombia.
\end{abstract}

Keywords

Border Policy, Colombia, Nicaragua, Borderline Difference, International Law, International Court of Justice. 


\title{
Colômbia e sua política de fronteira: desafios e perspectivas à luz do litígio com Nicarágua perante a Corte Internacional de Justiça
}

\author{
Diana María Angulo Cardona \\ María Isabel Cadrazco Saavedra \\ Alexander Pinzón Flórez.
}

\section{RESUMO}

Ao longo de 210 anos de vida republicana, o Estado colombiano perdeu e cedeu parte do território nacional em relação ao tamanho do Vice-Reino de Nova Granada. Dos 1.572 .248 km2 de território que pertenciam ao Vice-Reino, apenas $1.147 .748 \mathrm{~km} 2$ correspondem ao atual território nacional. Ou seja, o país perdeu cerca de 504.500 km2 ou 579.500 km2 se somarmos o território perdido devido ao contencioso marítimo com a Nicarágua. Dada a inexistência de políticas públicas de Estado que respondam de maneira efetiva e eficiente à defesa dos interesses nacionais no território insular colombiano, a decisão de 2012 da Corte Internacional de Justiça de Haia contra a ação movida pela Nicarágua contra a Colômbia em reivindicação de soberania sobre o Arquipélago, acrescentou $75.000 \mathrm{~km} 2$ a mais à perda territorial histórica que o país sofreu. Este artigo analisa os desafios da política de fronteira da Colômbia após a Constituição de 1991, o contexto social e político em que os dois últimos governos nacionais implementaram essas políticas na Ilha e o impacto que a decisão da CIJ teve sobre a redefinição não só da fronteira marítima colombiano-nicaraguense, mas também de toda a política de fronteira da Colômbia. 


\title{
La Colombie et sa politique frontalière: défis et perspectives à la lumière du différend avec le Nicaragua devant la Cour Internationale de Justice
}

\author{
Diana María Angulo Cardona \\ María Isabel Cadrazco Saavedra \\ Alexander Pinzón Flórez
}

\section{RÉSUMÉ}

Tout au long de ses 210 ans de vie républicaine, l'État colombien a perdu et cédé une partie du territoire national par rapport à la taille de la vice-royauté de Nouvelle-Grenade. Sur les 1572 248 km2 de territoire qui appartenaient à la vice-royauté, seuls 1147748 km2 correspondent au territoire national actuel. En d'autres termes, le pays a perdu quelque 504500 km2 ou 579500 km2 si l'on ajoute le territoire perdu en raison du différend maritime avec le Nicaragua. En l'absence de politiques publiques de l'État qui répondent de manière efficace et efficiente à la défense des intérêts nationaux dans le territoire insulaire colombien, l'arrêt de la Cour internationale de justice de La Haye en 2012 contre la plainte déposée par le Nicaragua contre la Colombie revendiquant la souveraineté sur l'archipel, a ajouté 75000 km2 supplémentaires à la perte territoriale historique que le pays a subie. Cet article analyse les défis de la politique frontalière de la Colombie après la Constitution de 1991, le contexte social et politique dans lequel les deux derniers gouvernements nationaux ont mis en œuvre ces politiques sur l'île et l'impact que l'arrêt de la CIJ a eu sur la redéfinition non seulement de la frontière maritime entre la Colombie et le Nicaragua, mais aussi de toute la politique frontalière de la Colombie.

\section{Mots-clés}

Politique frontalière, Colombie, Nicaragua, différend frontalier, droit international, Cour internationale de justice. 


\section{INTRODUCCIÓN}

La política exterior se entiende como aquella rama de la política de los Estados encargada de establecer la acción que estos despliegan ante los demás actores del Sistema Internacional. Esta no debe ir alejada de la política interna, pues como decisión de tipo estatal tiene que responder a los intereses nacionales. A lo largo de su corta historia como Estado-nación, Colombia ha experimentado varias transformaciones a nivel territorial, pues ha perdido gran parte de su superficie a favor de diversos países, como es el caso de la cesión de unos 350.000 km² que hoy son parte de Brasil y Perú.

Son dos los momentos más importantes en ese proceso de redefinición del territorio colombiano. El primero fue la separación de Panamá el 3 de noviembre de 1903, que representó una pérdida territorial de alrededor de $74.500 \mathrm{~km}^{2}$ y a su vez reconfiguró la identidad nacional que se había consolidado hasta ese momento. El segundo, aún más determinante que el anterior, sería la pérdida de $75.000 \mathrm{~km}^{2}$ de mar como consecuencia del fallo de la Corte Internacional de Justicia de La Haya del 19 de noviembre de 2012 a favor de Nicaragua. Este hecho obligó al Estado colombiano a repensar su política de fronteras, no a la luz de dinámicas de cooperación, sino mediante la coerción de un fallo judicial de obligatorio cumplimiento.

Tradicionalmente, la política exterior del Estado colombiano se ha caracterizado por ser de tipo gubernamental y no estatal. Cada cuatro años, con la llegada a la Casa de Nariño de un nuevo gobernante, cambian las prioridades en materia de política internacional. El único aspecto que quizá no ha perdido su vigencia y se mantiene pese a la variación de intereses relacionados con la política exterior es el centralismo del Estado a la hora de tomar decisiones eficientes de política pública en torno a las fronteras. Históricamente, la carencia de decisiones efectivas de carácter estatal y no de gobiernos de turno ha causado una sensación de abandono entre los habitantes de las regiones fronterizas del país, lo que se ha traducido en problemas sociales en el caso de la frontera colombo-venezolana o en sentimientos independentistas como en el caso de la frontera colombo-nicaragüense.

El presente artículo tiene por objetivo examinar los retos de la política de fronteras de Colombia respecto del fallo de la Corte Internacional de
Justicia de La Haya producto del diferendo entre el país y Nicaragua. Para esto se hace un análisis interpretativo de la política nacional de fronteras a partir de la Constitución de 1991. Posteriormente, se identifica el contexto social y político con base en las acciones de política pública de los gobiernos de Juan Manuel Santos e Iván Duque en torno a la Isla de San Andrés, Providencia y Santa Catalina. De igual manera, se indaga por la incidencia que el fallo de fondo de la CIJ tuvo sobre la formulación de la política fronteriza nacional, para concluir en una propuesta holística sobre los principales retos que el Estado colombiano enfrenta en términos de política pública de fronteras, es decir, una política exterior de fronteras interméstica que responda a los intereses nacionales e internacionales.

\section{METODOLOGíA}

Para responder a los interrogantes planteados se utilizó un enfoque metodológico cualitativo basado en la revisión de fuentes bibliográficas que permitieron analizar, en primer lugar, la política de fronteras de Colombia a partir de 1991 con la nueva Constitución colombiana. En segundo lugar, se identificaron las diferentes acciones de política pública que los últimos dos gobiernos de Colombia han desplegado en San Andrés, Providencia y Santa Catalina, y se estableció el impacto que el fallo de la CIJ ha tenido en la definición de una política de fronteras en Colombia. Las limitaciones del presente estudio radican, sin embargo, en la escasa información de tipo estatal sobre las acciones de política de fronteras aplicada en Colombia respecto de la República de Nicaragua, lo que abre la puerta para futuros análisis de la incidencia del mismo fallo, pero desde el lado nicaragüense.

\section{RESULTADOS Y DISCUSIÓN}

\section{Política fronteriza de Colombia a partir de 1990}

Tras el proceso de descolonización que tuvo Colombia a comienzos del siglo XIX, especialmente entre 1810 y el comienzo del periodo de Independencia y 1819 y el proceso de cierre de esta, una de las herencias más importantes al haber sido colonia española fue, justamente, el ordenamiento territorial (Borda, 2019). El principio de uti possidetis iuris fue pieza clave para la consolidación del proceso de formación del recién creado Estado-nación, no solo para el territorio colombiano, sino también para las 
demás colonias del Imperio español. No obstante, este mismo principio, junto con el complejo manejo geográfico y la "imprecisión de los mapas y la vastedad de las zonas limítrofes deshabitadas ocasionaron multitud de conflictos, a los cuales no ha sido ajeno nuestro país" (Ceballos \& Sanín, 2013, p. 24). Y en la lucha por la consecución de una nueva identidad que brindara autonomía y características esenciales a la nueva nación (pueblo, territorio y gobierno), Colombia y países como Venezuela, Brasil y Perú no tardarían en sumarse a la carrera por la delimitación territorial y todo lo que esto connotaba, incluidos conflictos entre las partes.

Ante tales imprecisiones y las actuales debilidades que implica el no tener una política de Estado robusta y bien fundamentada, según los autores mencionados anteriormente, desde lo heredado del Virreinato de la Nueva Granada $\left(1.572 .248 \mathrm{~km}^{2}\right)$ y el actual territorio nacional $\left(1.147 .748 \mathrm{~km}^{2}\right)$, Colombia ha ido perdiendo y cediendo territorio, alrededor de unos $484.500 \mathrm{~km}^{2}$ y $504.500 \mathrm{~km}^{2}$ (Ceballos \& Sanín, 2013, p. 31).

Ahora bien, la reflexión en el presente artículo, y de acuerdo con este breve repaso de las desavenencias que ha vivido el país históricamente con una actitud pasiva y resignada frente a una política fronteriza que responda y fortalezca los intereses nacionales, gira en torno a la necesidad de reconocer los fundamentos de una política de fronteras más robusta y que se sobreponga en el espacio y en el tiempo. Para tal efecto, se hace indispensable hacer un breve recuento de las principales y más recientes disposiciones de política pública en materia de fronteras.

Hasta 1991, en términos de legislación, con la Ley 10 de 1983, el Estado le confería al Ejecutivo los lineamientos para el manejo de la política de fronteras. Es decir, se sentarían las bases para que en la Constitución de 1991 se ratificara el manejo de la política fronteriza del Estado en manos del Ejecutivo. Así mismo, la ley facilitó el reconocimiento de las unidades territoriales que conforman las fronteras como regiones geopolíticamente estratégicas, sujetas al desarrollo económico, político y social.

Uno de los avances fundamentales en la definición de nuevas legislaciones para las fronteras ha sido la Constitución de 1991, pues esta dicta que es competencia del Ejecutivo dirigir las relaciones internacionales y formular la seguridad en términos de política exterior de la República. Según el artículo
9, las relaciones exteriores del Estado colombiano se fundamentan en la soberanía nacional, en el respeto a la autodeterminación de los pueblos y en el reconocimiento de los principios del derecho internacional aceptados por Colombia. Uno de los sus objetivos primordiales se orienta hacia la integración de Latinoamérica y el Caribe. En la Constitución Política de Colombia se estipula que el Estado "cooperará con otras naciones en la protección de los ecosistemas situados en las zonas fronterizas" (C. P., 1991, art. 80). La Carta Magna, en su ánimo de fortalecer una política de Estado en sus fronteras, definió no solo límites territoriales, sino que fue más allá y también abordó las fronteras espaciales, es decir, la órbita geoestacionaria y el espectro electromagnético.

En su artículo 337, la Constitución dejó sentadas las bases para la creación de una legislación especial para las regiones que conforman las zonas fronterizas. Además, desde el nuevo marco constitucional de 1991 se estableció que los diferentes entes territoriales podían articular programas de cooperación e integración a nivel regional y fronterizo. Lo anterior se dio con el fin de salvaguardar intereses en común y más allá de las mismas fronteras.

En 1995 se expidió la Ley 191, que se ha convertido en una de las iniciativas más robustas y claves en Colombia en materia fronteriza. Su objetivo era establecer un régimen especial para las zonas de frontera, con el fin de promover y facilitar su desarrollo económico, social, científico, tecnológico y cultural, tanto con las otras partes del país como con los países vecinos.

A partir de dicha ley y hasta el año 2004 se dispusieron algunas medidas en términos de política fronteriza que permitieron la creación de las Ilamadas Zonas de Integración Fronteriza, una con Ecuador y otra con Perú. Con el alcance de estas medidas también se logró tener en cuenta aspectos como la regulación de la calidad de combustible en las zonas fronterizas, la exención del pago de gravámenes arancelarios y la consecución de recursos para un fondo económico que modernizara las zonas de frontera, entre otros aspectos clave en dichos territorios.

Otras leyes sucedieron a la Ley de 1995. Tal es el caso de la Ley 677 de 2001, que apuntó a la normalización y creación de las famosas Zonas Económicas de Exportación en algunos municipios como 
Valledupar, Cúcuta y Buenaventura. La Ley 681 de 2001 creó el régimen de concesiones de combustibles en las zonas de frontera y definió el papel que jugarían entidades estatales como Ecopetrol en la distribución de combustibles en departamentos y municipios ubicados en zonas de frontera. La Ley 843 de 2003 reconoció las condiciones necesarias para el aprovechamiento de áreas naturales pertenecientes a zonas de frontera, con el fin de garantizar la protección del territorio nacional poblado por comunidades indígenas.

Ahora bien, siendo la Ley 191 de 1995 la columna vertebral de la legislación colombiana en materia fronteriza, el Gobierno colombiano ha creado algunos documentos Conpes que recogen diferentes avances en su implementación. Por ejemplo, el Conpes 3155 de 2002 brindó los lineamientos jurídicos encaminados al desarrollo de la política de integración y desarrollo fronterizo, y el Conpes 3272 de 2004 estableció los requisitos para que las entidades territoriales ubicadas en zonas de frontera accedan a los proyectos de infraestructura vial de la cual pueden ser beneficiarias.

Por su parte, el Decreto 1182 de 1999 le dio potestad al Ministerio de Relaciones Exteriores para la administración de las fronteras, por lo cual este creó la Dirección de Integración y Desarrollo Fronterizo, concediéndole funciones para la formulación de la política de fronteras y programas y proyectos para promover el desarrollo de las zonas fronterizas. Desde este marco emergen la Comisión Intersectorial de Integración y Desarrollo Fronterizo (CIIDEF), los Comités Territoriales Fronterizos y las Comisiones de Vecindad e Integración Fronteriza.

En el año 2014 se expide el documento Conpes 3805, que ha sido uno de los avances más recientes en la consolidación y el fortalecimiento de "la Política Pública de Prosperidad para las Fronteras destinada a generar unas fronteras más incluyentes, sostenibles y seguras; garantizando su desarrollo socioeconómico a través de la implementación de estrategias específicas y el aprovechamiento de sus potenciales endógenos" (Conpes 3805, 2014).

Las principales características de este documento se centran en fortalecer la institucionalidad del Gobierno Nacional y las entidades territoriales para la gestión del desarrollo, la integración fronteriza y la soberanía nacional, reducir las brechas socioeconómicas de los territorios fronterizos con relación al resto del país, promover condiciones que permitan el crecimiento sostenible de las regiones de frontera, integrar los territorios fronterizos entre sí, con la nación y países vecinos, y fortalecer la identidad, la cosmovisión y la organización social y política de los pueblos y comunidades indígenas, afrodescendientes, raizales y rom.

Ahora bien, aunque han existido políticas públicas de frontera que abordan necesidades en lo relacionado con aspectos económicos, sociales y culturales del Archipiélago de San Andrés, Providencia y Santa Catalina, su poca efectividad da a entender que lo sucedido con el diferendo marítimo entre Nicaragua y Colombia se dio en circunstancias de desfavorabilidad para el Estado colombiano, ante la ausencia de una política de Estado robusta sobre las fronteras. Como lo manifiestan Ceballos y Sanín (2013), una actitud de resignación ha llevado a que el actual territorio nacional pierda alrededor del $31 \%$ si se compara con lo que fue el territorio del Virreinato de la Nueva Granada.

\section{Acciones de política pública del Estado colombiano respecto del departamento de San Andrés, Providencia y Santa Catalina desde el año 2002}

El interés por estudiar y analizar las políticas públicas se debe a que el Estado responde a las demandas de la sociedad a través de estas. Como lo afirma Deubel (2003), "la aparición de un campo específico y el interés para analizar el objeto 'políticas públicas' se debe al crecimiento del intervencionismo del Estado en muchos aspectos de la vida en nuestras sociedades, independientemente de sus niveles de desarrollo" (p. 115). Para este autor, las políticas públicas activan el accionar político del Estado.

Las políticas públicas, al ser instrumentos de intervención del Estado, tienen su propio ciclo de vida. En primer lugar, aparecen cuando logran ser incluidas en la agenda, es decir, los problemas sentidos socialmente quedan contemplados dentro de la agenda pública del Gobierno. Una vez se surte este primer paso, viene el ciclo de la formulación de la política pública, en la cual se determina el problema a solucionar, de tal manera que esto le permite al Gobierno fijar dentro de su plan de desarrollo programas, proyectos y recursos que den respuesta a la problemática. Posteriormente, al hacerse ese reconocimiento, se estructura la implementación, donde se materializa la voluntad política 
del gobernante, la política pública se acciona y se vuelve un hecho político. Finalmente, en la fase de evaluación se perfecciona la política y se da lugar a la aparición de nuevos problemas que dejan como efecto nuevas políticas públicas.

A continuación, se hace una breve descripción y diagnóstico de la isla: está ubicada al noroccidente de Colombia, en la región Caribe insular; tiene una población de más de 79.000 habitantes según el último censo realizado en Colombia (DANE, 2018), y de estos el 72 \% vive en la zona urbana; el archipiélago ha venido experimentando varios periodos de crisis económica, social e institucional, los cuales se ven reflejados en los altos índices de pobreza multidimensional, que oscila en un 39,8\%, mientras el promedio nacional se sitúa en un $27 \%$.

Los distintos gobiernos nacionales han hecho esfuerzos de inversión con recursos financieros y técnicos. Sin embargo, este apoyo no ha generado una mayor capacidad de respuesta y de desarrollo a largo plazo. Entre las causas principales se destacan: problemas asociados a una crisis en el sector de la economía, concentrada en un solo sector y con poca diversificación de mercados, un mínimo esfuerzo fiscal por parte del departamento, una tasa de desempleo del $25 \%$, una tasa de informalidad del $90 \%$, una tasa de analfabetismo del $14,3 \%$, una tasa de deserción escolar del $40 \%$ y un manejo inadecuado de los recursos públicos. Esta degradación económica ha traído como consecuencia la inconformidad de los grupos raizales y un alto grado de marginalidad de la comunidad en general.

Con base en los documentos Conpes 3058 de 1999 y 3805 del 2014, y los documentos institucionales Informe al Congreso 2008, del Gobierno del expresidente Álvaro Uribe, y San Andrés y Providencia. Ruta del desarrollo sostenible, de la Banca de Desarrollo Territorial (Findeter, 2019), se hace un breve recuento de las principales políticas públicas que se han implementado y se implementarán en la isla:

En primer lugar, se identifica que San Andrés y Providencia cuentan con una producción muy concentrada, con fuertes bases en una economía terciaria. Lo anterior, se evidencia en la participación del sector de comercio, que abarca cerca del $60 \%$ del total de la producción del departamento. (Findeter, 2019, p. 15).
En el caso del turismo, se presenta una deficiente infraestructura hotelera y de transporte, así mismo un rezago en servicios como recreación, gastronomía y deportes. También es preocupante el deterioro de los atractivos turísticos tradicionales que caracterizan a la Isla.

Frente al empleo y la pobreza rural, de acuerdo con el censo poblacional, la población en edad de trabajar se encuentra en condición inactiva y se evidencian problemas de pobreza y competitividad en la ruralidad. Por un lado, la baja productividad; y por el otro, la alta concentración de la propiedad de la tierra.

En el sector de competitividad e innovación, se destaca por una alta calidad en la producción de conocimiento y tecnología, especialmente en el sistema de salud para la primera infancia y en innovación empresarial (Findeter, 2019). Sin embargo, la cobertura en conectividad a internet es muy deficiente y cuenta con una banda ancha muy lenta. El acceso a los servicios de internet en San Andrés es del 2,67 \% frente a un promedio nacional del $8,67 \%$.

En la gestión administrativa, San Andrés se caracteriza por un bajo desempeño, debido a la deficiente capacidad técnica de los funcionarios públicos, a problemas de corrupción y a una alta injerencia política. En el ámbito de la infraestructura social y particularmente en agua y alcantarillado, es preocupante la baja cobertura de servicios públicos, donde la isla ocupa el puesto 21 con un puntaje de 6,73 sobre 10 . Más de la mitad de sus habitantes no cuentan con acceso a alcantarillado (81\%), agua potable (30 \%) ni vivienda. Además, existe un déficit de producción de agua potable, lo cual ocasiona racionamiento.

En el sector salud, uno de los mayores rezagos es la alta tasa de mortalidad infantil, debido a las condiciones nutricionales de la madre y los menores. Lo anterior se suma a condiciones de saneamiento básico, acceso a agua potable y a servicios de salud. Así mismo, en cuanto al acceso al servicio de salud, San Andrés presenta una dilación en la afiliación de la población vulnerable al Sistema General de Seguridad Social, ubicándose en el $85 \%$ frente al promedio nacional, que se encuentra en el 98,85\%. Las debilidades institucionales del sector salud se reflejan en la inadecuada vigilancia 
y control social sobre el desempeño del régimen subsidiado, la inoperatividad del Consejo Territorial de Seguridad Social en Salud, el inadecuado proceso de aseguramiento y las dificultades para definir la red de servicios del primer nivel de atención en el departamento.

Por su parte, el sistema educativo presenta una escasa oferta de educación superior y de bibliotecas públicas con óptimas condiciones de conectividad. Tampoco existe articulación entre la educación básica, media y superior con el contexto pluricultural y bilingüe y con el sector productivo. La tasa de analfabetismo para la población de 15 años en adelante es del $14,3 \%$, superior a la media nacional, que se encuentra en $8,4 \%$, y la tasa de deserción escolar es del 4,82\%, mientras que la nacional se ubicó en 4,28 \%. En cuanto a la cobertura en educación superior, el indicador se encuentra en $20,4 \%$, mientras la cobertura promedio nacional es del $33,3 \%$.

En vivienda hay un déficit habitacional que duplica la media nacional tanto en la zona urbana como en la rural: en primera es del $37 \%$, mientras a nivel nacional es del $27 \%$, y en la segunda es del $85,7 \%$, mientras a nivel nacional está en el $68,3 \%$. Debido a la condición geográfica de la isla, la energía eléctrica no está conectada al Sistema Interconectado Nacional, lo cual incrementa el valor del servicio, dificulta la cobertura y entorpece su prestación, dado que está sujeto a horarios y restricciones.

De otra parte, se presenta una inseguridad fronteriza marcada por "las limitaciones en el manejo estratégico al tema fronterizo y en la coordinación interinstitucional, así como en las capacidades de la fuerza pública para controlar los límites" (Conpes 3805, 2014). Las principales amenazas son el narcotráfico, el contrabando y la delincuencia común. Teniendo en cuenta la ley de régimen especial para fronteras (Ley 191 de 1995) y los documentos institucionales antes mencionados, se hace referencia a las principales políticas públicas que se han implementado para contrarrestarlas.

En turismo, los gobiernos le han apostado a un plan maestro que convierta a San Andrés en destino turístico internacional (turismo deportivo, apertura de vuelos comerciales, recuperación del patrimonio arquitectónico tradicional), cuyos ejes principales sean el respeto y la promoción de la riqueza cultural y la compatibilidad con el medio ambiente. Así mismo, en asocio con Findeter y teniendo en cuenta que Colombia ha venido experimentando un crecimiento en la exportación de bienes y servicios creativos, el actual Gobierno viene liderando el impulso de las industrias creativas y culturales "mediante un sistema de asistencia técnica, financiación y ejecución de proyectos innovadores" (Findeter, 2019, p. 19), mejor conocido como "economía naranja".

En política de educación y TICS, la estrategia se ha enfocado en ampliar la cobertura en educación básica primaria, secundaria, técnica, tecnológica y superior en el marco del programa Jóvenes en Acción, así como en garantizar la conectividad mediante la conexión a fibra óptica, la instalación de los kioscos Vive Digital y el programa Computadores para Educar, como parte de la alfabetización digital. También se implementó un proyecto de etnoeducación, plurilingüe e intercultural, y se institucionalizó la Cátedra Afrocolombiana, de tal manera que la educación esté acorde con el contexto sociocultural del departamento. Adicionalmente, se promueve la formación de licenciados plurilingües que además de inglés y español dominen las lenguas nativas.

En materia de empleo se ha apuntado a establecer condiciones que aumenten las posibilidades del desarrollo económico, disminuyendo los costos de producción y transacción (a través de instrumentos como la exenciones impositivas, incentivos tributarios para la creación de nuevas empresas, capital semilla, establecimiento de zonas francas, producción regional de insumos y energía, entre otras), de modo que se incremente la inversión pública y privada en los renglones que presentan ventajas comparativas y por ende se dinamice el mercado laboral. La política también busca fortalecer un observatorio de empleo que articule la información sobre oferta y demanda de puestos de trabajo. De igual manera, se han abordado estrategias de empleabilidad como el mantenimiento de vías con participación comunitaria, el fomento a la microempresa turística mediante la capacitación de los microempresarios en actividad turística y la modernización de la actividad pesquera con transferencia de tecnología.

En empleo rural se vienen priorizando proyectos de desarrollo agropecuario y rural enfocados a la sustitución de cultivos ilícitos (granjas porcícolas, artesanías, proyectos productivos agroindustriales). Frente al empleo en el campo, el Gobierno lidera 
una iniciativa que pretende generar un modelo de desarrollo comercial agrícola en los espacios periurbanos (es decir que están situados es la periferia de la ciudad), a partir de la comercialización de los productos en la isla y sin intermediarios.

Las políticas en salud han tenido dos enfoques: uno que se concentra en mejorar la infraestructura y la red hospitalaria, y el otro en la calidad de la atención y la prevención. En infraestructura se han invertido recursos en adecuación de la planta física del hospital departamental Timothy Britton, los puestos y centros de salud y la construcción de un laboratorio de salud pública departamental. En cuanto al servicio de salud, se propone la formulación e implementación del plan de atención básica, con una ampliación y renovación de afiliación al régimen subsidiado.

En el ámbito de fortalecimiento institucional, la política ha girado en torno a trabajar por mejorar las capacidades de planificación territorial y una debida articulación interinstitucional con los diferentes niveles del Gobierno Nacional, que conlleve a una mayor presencia del Estado. Para ello se viene capacitando a los funcionarios públicos a través de la Escuela Superior de Administración Pública (ESAP). Del mismo modo, se viene financiando un programa de lucha contra la corrupción, con el fin de identificar los focos institucionales del flagelo y crear instrumentos que faciliten la transparencia, el seguimiento y el control ciudadano.

Por otra parte, los gobiernos han implementado un esquema público-privado que preste y mejore la eficiencia y la calidad de los servicios de electricidad, agua, alcantarillado y disposición de residuos sólidos. También se busca fortalecer capacidades para generar energía renovable, desarrollando energía eólica, energía solar y energía térmica del océano.

En vivienda, particularmente, se ha apuntado a mejorar las viviendas existentes y a incentivar programas de construcción de vivienda nueva. Actualmente se pretende generar un proyecto de desarrollo urbano de viviendas bioclimáticas con conectividad y acceso a servicios públicos.

Finalmente, la política de seguridad se ha contemplado desde varios ejes: seguimiento al delito, aumento del pie de fuerza de la Policía Nacional para que se dediquen a la policía comunitaria, construcción de estaciones de policía, incremento del control del tráfico internacional fortaleciendo la coordinación entre entidades nacionales e internacionales por el paso fronterizo y, por último, la inclusión del gobernador y el alcalde dentro de la institucionalidad creada en la "ley anticontrabando", a fin de coordinar acciones que permitan combatir este fenómeno.

Se puede señalar que la soberanía que debe ejercer el Estado colombiano sobre la San Andrés, Providencia y Santa Catalina va más allá de la presencia de las fuerzas militares en el territorio. Actualmente, a causa de la pandemia, la isla se encuentra en lo que el primer gobernador de San Andrés, Kent Francis (1991), denomina un "aneurisma social". Y el único camino que evitará que este aneurisma estalle es la salida institucional que se debe dar a esta crítica situación, robusteciendo la presencia estatal con políticas públicas que permitan reactivar la economía, no solo desde el turismo y el comercio, sino también desde otros sectores como el agrícola (producción de alimentos), el ambiental (preservación y cuidado el medio ambiente) y la producción de energías alternativas. Así mismo, urge adoptar estrategias de intervención social que se ajusten a las características insulares del territorio y a su posición geoestratégica.

\section{Incidencia del fallo de la Corte Internacional de Justicia de 2012 sobre la política de fronteras de los últimos dos gobiernos de Colombia}

Entender la dinámica fronteriza de Colombia implica revisar hechos históricos que han determinado su relación con los vecinos. La pérdida de Panamá, la guerra con el Perú, el conflicto armado interno y, recientemente, el diferendo limítrofe con Nicaragua han marcado los 210 años de vida republicana del país en lo que a sus relaciones de vecindad se refiere. Para Socorro Ramírez (2006, p. 30), "las Fronteras Nacionales son más que los límites terrestres, y están definidas por las interacciones poblacionales; ambientales; comerciales y de seguridad". Y es quizá en el exiguo entendimiento de las diferentes dinámicas fronterizas en lo que ha fallado el Estado Colombiano a la hora de definir una política estatal de fronteras.

Como se mencionó anteriormente, el artículo 9 de la Constitución Política colombiana de 1991 establece que uno de los principios orientadores de la política exterior del país es la integración con 
América Latina y el Caribe, ya que se considera a Colombia como parte de lo que se denomina "El Gran Caribe", esa zona geográfica que abarca desde Quintana Roo en México hasta Salvador de Bahía en el Brasil, pasando por las Antillas Mayores y Menores, Venezuela y Colombia.

El Tratado Esguerra Bárcenas, firmado en 1928 y que define los límites entre la República de Colombia y la República de Nicaragua, es un texto que abarca aspectos fundamentales para la comprensión de las dinámicas limítrofes entre ambos países: por un lado, se reconoce la soberanía de Nicaragua sobre la costa Mosquitia y la soberanía de Colombia sobre la isla de San Andrés, Providencia y Santa Catalina, y por otro lado, se aclara la no vinculación de algunos cayos como Serrana, Serranilla, Roncador y Quitasueño, ya que al momento de firmar el tratado se encontraban en litigio entre Colombia y los Estados Unidos de América.

No fue sino hasta 1980, con la promulgación del Libro Blanco de Política Exterior nicaragüense, y después en 1990, cuando el Estado de Nicaragua, representado en el Gobierno de Violeta Barrios de Chamorro, decidió desconocer el Tratado Esguerra Bárcenas de 1928 y reclamar para su país la soberanía de San Andrés, Providencia y Santa Catalina, así como la de los cayos Serrana, Serranilla, Roncador y Quitasueño y demás islas e islotes adyacentes, argumentando que al momento de la firma del mencionado tratado el país centroamericano se encontraba, tal y como lo menciona Alvarado (2014, p. 266), "invadido y dominado por una política nacional e internacional liderada desde los Estados Unidos, aliado tradicional de Colombia".

Así, el Gobierno de Barrios de Chamorro y los siguientes del régimen sandinista, actualmente en el poder en el vecino país, resolvieron no solo desconocer la legitimidad de aquel tratado, sino también la validez de la Real Orden de 1803 expedida por el Reino de España, donde se reconoce que el Virreinato de la Nueva Granada, hoy Colombia, tiene soberanía plena sobre San Andrés, Providencia y Santa Catalina y los demás cayos e islotes adyacentes. De esta manera, siguiendo una política exterior de Estado establecida en 1980 con la promulgación del Libro Blanco antes mencionado, el 6 de diciembre de 2001 Nicaragua presentó una demanda formal ante la Corte Internacional de Justicia con sede en La Haya (Países Bajos), en la que reclamaba, por un lado, la soberanía total sobre San Andrés, Providencia y Santa Catalina y los demás cayos e islotes adyacentes, y, por otro, la delimitación de la frontera marítima entre ambos países, la zona económica exclusiva y la plataforma continental.

A partir de ese momento y durante más de 12 años, ambos países argumentaron ante la CIJ diferentes posiciones que trataron de validar sus pretensiones respecto del diferendo limítrofe. Por su lado, Nicaragua se acogió a la Convención Internacional del Derecho del Mar (Convemar) de 1982 -de la que Colombia no hace parte- para justificar sus pretensiones ante la Corte. Entretanto, la defensa de Colombia país argumentaba, según lo especifica Augusto Ramírez Ocampo (2009), que aunque el país no hubiese ratificado la Convemar de 1982, es respetuoso con el derecho internacional público y que el principio de uti possidetis iuris es aplicable para reclamar la soberanía de un Estado sobre un determinado territorio. Respecto a este tema, Gálvez (2009) insiste en que el uti possidetis iuris "puede ayudar a delimitar las fronteras en un diferendo limítrofe, puesto que la costumbre, como principio de derecho internacional público, es ampliamente aceptada por la comunidad internacional" (p. 10).

De acuerdo con Rafael Nieto (2014), la estrategia de defensa de Colombia ante la Corte Internacional de Justicia abarcó cuatro aspectos principales: i) se demandó la adhesión del país al Pacto de Bogotá, que define los mecanismos de resolución de conflictos limítrofes para el país; ii) se declaró una zona contigua integral que consolida la unidad territorial del Archipiélago; iii) se consagró la Reserva Sea Flower como patrimonio de la humanidad por parte de la Unesco, reafirmando la importancia de esta zona geográfica para el país, y iv) Colombia argumentó que la soberanía de San Andrés, Providencia y Santa Catalina, además de respetar el uti possidetis iuris mencionado anteriormente, defiende al continente de las ambiciones imperialistas del gobierno de Daniel Ortega Saavedra y el sandinismo en la región.

Así pues, la Corte Internacional de Justicia (2012) emitió dos fallos sobre el tema, en los que dio respuesta a las pretensiones de Nicaragua y Colombia. En el primero, pronunciado en 2007, se declaraba competente para emitir un fallo de fondo sobre dos cuestiones: por un lado, la soberanía de San Andrés, Providencia y Santa Catalina y, por el otro, la delimitación marítima de ambos países. En el segundo, de 2012, sentenciaba que Colombia ejercía 
soberanía sobre la isla de San Andrés, Providencia y Santa Catalina, así como sobre los cayos e islotes adyacentes. Adicionalmente, la CIJ resolvió la delimitación de la plataforma continental de ambos países de acuerdo a lo establecido en la Convemar de 1982, que a pesar de no haber sido ratificada por el Estado colombiano, tiene todas las normas y principios del derecho internacional público, lo que la hace competente para conocer el caso.

Apenas se supo de la decisión -que era de inmediato cumplimiento, de única instancia e inapelable, contrario a lo que sucede en el derecho nacional-, el Estado colombiano, en cabeza de Juan Manuel Santos Calderón (2010-2018), invitó al Gobierno de Nicaragua a aplicar el fallo mediante la suscripción de un nuevo tratado limítrofe, pues es la única forma de definir las fronteras del país, de acuerdo con la Constitución y las leyes colombianas. No obstante, el Gobierno de Managua y el régimen de Daniel Ortega se han negado en múltiples oportunidades a la propuesta.

Tanto el Gobierno de Santos como el de su sucesor, Iván Duque, hicieron múltiples promesas a la población de San Andrés, Providencia y Santa Catalina, un departamento tradicionalmente olvidado y alejado de la realidad política que se vive en Bogotá. Hasta el momento, como lo señala la comunidad raizal sanandresana, dicas promesas "han sido incumplidas al igual que la lucha por los intereses de los pobladores de San Andrés" (Ramírez, Mantilla \& Román, 2020 p. 49). Tan es así que, según Araiza (2009), aún antes del fallo de la CIJ y de las medidas políticas que desde la institucionalidad del Estado colombiano se tomaron respecto del departamento de San Andrés, Providencia y Santa Catalina, cerca del $42 \%$ de la población de la isla no se sentía ni colombiana ni nicaragüense, y prefería ser un Estado independiente que jalonara su propio desarrollo.

Actualmente, existe ante la Corte Internacional de Justicia de La Haya una segunda demanda de Nicaragua contra Colombia, radicada el 16 de septiembre de 2013, que solicita al organismo internacional determinar definitivamente la plataforma continental y los límites marítimos entre los dos Estados. Colombia, pese a haber renunciado al Pacto de Bogotá para argumentar que la CIJ no era competente para revisar el caso, se notificó, designó agentes y concurrió a la primera audiencia sobre este nuevo caso, lo que le otorgó inmediato reconocimiento a la Corte para conocer y fallar sobre este nuevo caso dentro del diferendo.

Así las cosas, se puede ver que el Estado colombiano no modificó su política de fronteras después del fallo de 2012, ya que tradicionalmente su política exterior ha tenido un enfoque de gobierno y no de Estado, y por tanto esta se maneja desde Bogotá teniendo en cuenta la agenda política del Gobierno de turno y desconociendo las particularidades políticas, económicas, ambientales, sociales y culturales de las zonas de frontera terrestre y marítima del país.

\section{Propuesta holística para una política fronteriza de Colombia a la luz del fallo de la Corte Internacional de Justicia de 2012}

Desde que inició su aventura republicana, Colombia se ha caracterizado por ser un país con una política exterior cambiante que responde a los intereses de las élites en el poder y de aquellos grupos de influencia que las rodean y que se han mantenido durante más de 200 años. Quizá el único principio de política exterior que todos los gobiernos colombianos han aplicado a lo largo de los dos siglos de historia como Estado-nación es aquel que promueve el respeto por el derecho internacional público como herramienta de diplomacia para el país. En efecto, históricamente y dentro del respeto al derecho internacional público, Colombia ha hecho del uti possidetis iuris un principio fundamental de política exterior acorde a las leyes internacionales, dotando a sus diplomáticos de herramientas jurídicas en el campo del derecho internacional que puedan defender al país (Acevedo, 2017).

Es imperativo, entonces, que Colombia defina una política exterior de Estado, como lo recomendó la Misión de Política Exterior liderada por FedesarroIlo en 2010, la cual debe responder no solo a los intereses de los principales hegemones globales en el poder, sino también a los intereses domésticos, de tal manera que pueda aglutinar en torno a una noción de país a todos los sectores nacionales, sean de corte público o privado, y dentro de estos intereses por supuesto impulsar la política de fronteras e integración regional.

Para que haya una integración regional efectiva es necesario que existan vínculos de confianza entre los países de Latinoamérica y el Caribe. Por ello, el 
dinamismo de las fronteras nacionales es de vital importancia para la inserción de Colombia en el escenario regional. Sin embargo, las relaciones de integración regional en el continente, en el caso colombiano, han estado marcadas por la desconfianza de los países vecinos hacia el mismo país, producto de su inestable situación de seguridad.

Tras el fallo de la Corte Internacional de Justicia de La Haya en el año 2012, Colombia ha enfrentado nuevos retos en términos de política fronteriza. Por un lado, se hace necesario actualizar las políticas existentes, pero con un enfoque integral y diferenciado con el interior de la Nación y con las zonas fronterizas de los países vecinos. Por otro lado, se requiere articular una mayor capacidad institucional de las acciones en las fronteras para fortalecer aspectos como la resiliencia y disminuir las debilidades de las fronteras como casos aislados de la centralización del Gobierno Nacional.

La política de fronteras debe ser una política de Estado que permita un acuerdo a mediano y largo plazo sobre lo fundamental entre los actores públicos y privados que tienen presencia en la vida política, económica, social y cultural del país. Estos acuerdos implican, en primer lugar, una fuerte presencia estatal en la cual converjan el Gobierno Nacional, departamental, local y el sector privado, lo que fortalecerá la articulación interinstitucional y sus capacidades de planeación y gestión. En segundo lugar, conviene promover políticas públicas de inclusión desde la diversidad étnica y cultural que permitan el desarrollo y el crecimiento sostenible de las zonas de frontera. Por último, dentro de la política exterior del país se debe considerar a las zonas de frontera como territorios estratégicos que conlleven a la adecuada internacionalización de nuestra nación.

Otro aspecto importante en aras de reforzar la integración regional y las relaciones con los vecinos se ve reflejado en la nueva política colombiana hacia el Caribe, iniciativa que se ha visto reforzada desde que el historiador cartagenero Alfonso Múnera es el Secretario General de la Asociación de Estados del Caribe, foro de cooperación y diálogo político de los países de la Gran Cuenca del Caribe (Ramírez, 2011). Otra poderosa razón por la cual Colombia hizo su reingreso como actor regional al Gran Caribe fue, precisamente, el fallo de la CIJ en el litigio con Nicaragua que redefinió el límite fronterizo entre los dos países y despojó a Colombia de una gran cantidad de territorio marítimo. Quizá este duro golpe a la geografía y geopolítica colombiana ayude a los policymakers nacionales a entender que Colombia es un país caribe y que debe reconocerse y aceptarse como tal, pues desde tiempos de la Colonia ha compartido con Jamaica y Cuba, dos de los grandes países de la zona, vínculos políticos, históricos y culturales (González, 2006) que son imborrables de la memoria colectiva de los pueblos.

Colombia debe comprender que sus relaciones de vecindad, además de ser vistas como un asunto de seguridad nacional o de límites terrestres, deben ser estudiadas, comprendidas y formuladas desde un doble enfoque que abarque lo local y lo nacional. Es así como se atenderán las necesidades no solo del Estado nacional, que busca defender su territorio y soberanía desde el punto de vista del realismo clásico de las relaciones internacionales, sino también la construcción de lazos de identidades e intereses comunes con los vecinos, que ayuden a formular políticas públicas macro para las zonas de frontera y prioricen el beneficio de las comunidades de estas regiones, que al final de cuentas son las más beneficiadas o perjudicadas en momentos de crisis o prosperidad en las relaciones estatales entre los Estados fronterizos.

\section{CONCLUSIONES}

El estudio de las relaciones de vecindad es vital a la hora de comprender el diseño de la política exterior de un país y viceversa. Por eso, se hace necesario conocer y entender las doctrinas de política exterior nacional. Estos presupuestos teóricos son importantes para analizar desde el punto de vista de la integración regional cómo en la actualidad Colombia está construyendo unas relaciones de confianza con sus vecinos que han dado pie a avances significativos, como el retiro de la demanda que Ecuador había interpuesto ante la Corte Internacional de Justicia contra Colombia por el caso de las fumigaciones con glifosato en las zonas de frontera.

Desde la década de los noventa, los diferentes gobiernos de Colombia promulgaron una serie de documentos de política pública que han legislado sobre la política de fronteras, en conjunto con determinaciones particulares en lo relativo a la isla de San Andrés, Providencia y Santa Catalina, pero aun así el Estado colombiano no ha implementado desde el orden nacional una política de fronteras efectiva que responda, entre otros factores, al fallo 
de fondo de la Corte Internacional de Justicia de La Haya de 2012.

Aún quedan muchos retos para Colombia en relación a su inmersión en la región y al fortalecimiento de las relaciones con los vecinos. Por ejemplo, hay que llegar a una serie de acuerdos de cooperación con el Gobierno de la República de Nicaragua, que permitan en una doble vía, primero, el acatamiento del fallo de la CIJ y, segundo, el aseguramiento del derecho al trabajo de los pescadores de San Andrés Islas.
Otra tarea pendiente del Estado colombiano es involucrar a los países vecinos en el camino del posconflicto que el país va a empezar a transitar, pues la internacionalización del conflicto ha dado pie a que el vecindario también deba tomar medidas para prevenir la violencia que exporta Colombia. En este punto en particular es sumamente importante contar con el apoyo de Brasil como hegemón de la región y de los mecanismos del Consejo de Defensa Sudamericano, organismo que fue creado precisamente para hacer de Sudamérica una región no de paz negativa, sino de paz positiva. 
ÁREA DE DERECHO INTERNACIONAL Y RELACIONES INTERNACIONALES

* Colombia y su política de fronteras: retos y perspectivas a la luz del litigio con Nicaragua ante

la Corte Internacional de Justicia

\section{REFERENCIAS}

Acevedo Vélez, J. J. (2017). Del uti possidetis iure a la Corte Internacional de La Haya: la decadencia de la diplomacia colombiana en la defensa del territorio. Summa luris, 5(2), 269-295. Recuperado de https://www.funlam.edu.co/revistas/index.php/summaiuris/ article/view/2597

Alvarado, O. A. (2014). El conflicto fronterizo entre Colombia y Nicaragua: Recuento histórico de una lucha por el territorio. Historia Caribe, 9(25), 241-271. Recuperado de http:// investigaciones.uniatlantico.edu.co/revistas/ index.php/Historia_Caribe/article/view/1237

Araiza, R. (2009). Conflicto colombo-nicaragüense desde la perspectiva de la jurisprudencia de la Corte Internacional de La Haya. Cuadernos del Caribe, 7(12), 144-154. Recuperado de https://revistas.unal.edu.co/index.php/ ccaribe/article/view/41396

Banca de Desarrollo Territorial (Findeter). (2019). San Andrés y Providencia. Ruta del desarrollo sostenible. Recuperado de http://www.colombialider.org/wp-content/uploads/2020/04/ San-Andr\%C3\%A9s.pdf

Borda, S. (2019). ¿Por qué somos tan parroquiales? Bogotá: Planeta.

Ceballos, M. \& Sanín, N. (2013). La llegada del dragón. Bogotá: Panamericana.

Consejo Nacional de Política Económica y Social. (1999). Documento Conpes 3058: Estrategia del Gobierno nacional para apoyar el desarrollo del departamento Archipiélago de San Andrés, Providencia y Santa Catalina. Recuperado de https://colaboracion.dnp.gov.co/ CDT/CONPES/Econ\%C3\%B3micos/3058.pdf

Consejo Nacional de Política Económica y Social. (2014). Documento Conpes 3805: Prosperidad para las fronteras de Colombia.
Recuperado de https://colaboracion.dnp.gov. co/CDT/Conpes/Econ\%C3\%B3micos/3805. pdf

Corte Internacional de Justicia. Sentencia en la causa relativa a la controversia territorial y marítima (Nicaragua vs. Colombia), 19 de noviembre de 2012. Recuperado de https://www.cancilleria.gov.co/sites/default/files/litigio_nicaragua/PRINCIPALES\%20DOCUMENTOS/ traduccion_esp_sentencia_del_19_de_noviembre_de_2012.pdf

Gálvez, A. (2004). El Uti Possidetis luris y la Corte Internacional de Justicia. Revista de Derecho, 21 (21), 131-138. Recuperado de http://rcientificas.uninorte.edu.co/index.php/derecho/ article/view/2950/2029

González, R. (2006). Colombia en el Caribe. Revista Huellas, 76-77, 39-45. Recuperado de https:// guayacan.uninorte.edu.co/4PL1CACION35/ publicaciones/huellas/ebook/huellasno76_77/index.html

Nieto, R. (2014). Archipiélago de San Andrés y Providencia. Las estrategias de Colombia y la nueva demanda de Nicaragua ante la Corte Internacional de Justicia. Revista de la Facultad de Derecho, 5(2), 1-9. Recuperado de https://revistas.unc.edu.ar/index.php/ refade/article/view/11543/11975

Presidencia de la República de Colombia. (2008). Informe al Congreso 2008. Álvaro Uribe Vélez. Recuperado de https://colaboracion.dnp.gov. $\mathrm{co} / \mathrm{CDT} /$ Sinergia/Documentos/Informe_Presidente_al_Congreso_de_la_Republica_2008. pdf

Ramírez Ocampo, A. (2009). La decisión de la Corte Internacional de Justicia sobre las excepciones preliminares propuestas por Colombia en el caso Nicaragua contra Colombia. Cuadernos del Caribe, 1(12), 23-52. Recuperado 
de: https://revistas.unal.edu.co/index.php/ ccaribe/article/view/41386/42962

Ramírez, S. (2006). Colombia y Venezuela: ¿De una aguda tensión a una asociación estratégica? En S. Ramírez y J. Cadenas (Coords. y Edits.), Colombia-Venezuela: retos de la convivencia (pp. 23-57). Bogotá: Universidad Nacional de Colombia.

Ramírez, S. (2011). AEC: ¿De los Caribe al Gran Caribe? En H. J. Godoy, R. González y G. Orozco (Edits.), Construyendo lo global. Aportes al debate de relaciones internacionales (pp. 140-169). Barranquilla: Uninorte.

Ramírez, D. F., Mantilla, S. C. \& Román, R. (2020). Actores, motivaciones e intereses de la paradiplomacia en la frontera del Caribe insular colombiano después del fallo de La Haya. Estudios Políticos, 57, 42-69. DOI: 10.17533/ udea.espo.n57a03.

Röth, A. (2011). Introducción para el análisis de las Políticas Públicas. Cuadernos de Administración, 19(30), 113-128. DOI: 10.25100/cdea. v19i30.120 